\title{
ACCELERATION OF INTERSTELLAR CLOUDS BY O-TYPE STARS
}

\author{
JAN H. OORT* AND LyMan SPITZER, JR. \\ Leiden Observatory and Princeton University Observatory \\ Received June 18, 1954
}

\begin{abstract}
A possible mechanism is proposed for the acceleration of interstellar clouds. The ultraviolet light from a newly born $O$ star heats the gas in a dense interstellar cloud from $100^{\circ}$ to $10,000^{\circ} \mathrm{K}$. The resultant increase in pressure leads to high accelerations, and in this way an appreciable fraction of the radiant energy from a hot star may be converted into kinetic energy of cloud motions.

Since a detailed treatment of this problem would be very intricate, involving an integration through time both of the hydrodynamic equations and of the equation of radiative transfer, a preliminary analysis is carried through. When the side of the cloud facing the $\mathrm{O}$ star is heated, the heated cloud gases expand into the less dense material between the cloud and the $O$ star. The momentum carried away by these gases is computed approximately, on the assumption that the pressure of the gas originally surrounding the cloud is negligible. The acceleration of the cloud is then found from the momentum carried away, with the use of Newton's third law of motion but with complete neglect of all detailed hydrodynamic effects within the cloud.

The analysis is applied to three physical situations: (a) formation of an $\mathrm{O}$ star near a single dense cloud, which then becomes accelerated; $(b)$ approach of a cloud to an $\mathrm{O}$ star in a steady state, with repulsion of the cloud by the "rocket effect" of the expelled gases; $(c)$ formation of an $\mathrm{O}$ star in an extended cloud complex of such large mass that the $\mathrm{O}$ star can ionize only part of it; the outside cool shell may then be driven away. In all three cases, the mass of the neutral cloud decreases during the interaction with the radiation field, and very high velocities may be attained. It seems possible to explain by such mechanisms the high velocities that are observed in the faint components of interstellar lines. It may be, even, that all random cloud motions in the interstellar gas originate in this way.
\end{abstract}

\section{INTRODUCTION}

The multiplicity of the interstellar $\mathrm{K}$ and $\mathrm{H}$ lines, investigated first by Beals (1936), then very extensively by Adams (1949), demonstrates that interstellar gas clouds have appreciable random motions with respect to one another. The distribution of cloud velocities has been considered by Whipple (1948), Blaauw (1952b), Searle (1952), and Schlüter, Schmidt, and Stumpff (1953) on the basis of the data by Adams. Whipple showed that the distribution was non-Maxwellian; he fitted the observations with two Maxwellian distributions: large clouds, with a root-mean-square radial velocity in the neighborhood of $8 \mathrm{~km} / \mathrm{sec}$, and small clouds, with a corresponding velocity of about $25 \mathrm{~km} / \mathrm{sec}$. In the analysis by Blaauw, which takes into account the overlapping of components produced in different clouds, the observations are fitted with a distribution function proportional to $\exp (-v / \eta)$, where $\eta$ is $5 \mathrm{~km} / \mathrm{sec}$; the corresponding root-meansquare velocity is $7 \mathrm{~km} / \mathrm{sec}$. However, neither of these two theoretical distributions provides a fit for the few clouds observed with very high velocities, between 50 and 100 $\mathrm{km} / \mathrm{sec}$. As shown by Spitzer (1948b), a root-mean-square random cloud velocity of $9 \mathrm{~km} / \mathrm{sec}$ is consistent also with the observed equivalent widths of the interstellar D lines. Searle and Schlüter, Schmidt, and Stumpff discuss the prevalence of negative values among the clouds with random velocities in excess of $15 \mathrm{~km} / \mathrm{sec}$, a problem that had also been indicated by Blaauw.

Observations of the hydrogen line near $21 \mathrm{~cm}$ give slightly higher random velocities. From extensive measures in the northern Milky Way, van de Hulst, Muller, and Oort (1954) have derived $\eta=8.5 \mathrm{~km} / \mathrm{sec}$. This value is in good agreement with the mean velocity found by Blaauw from his more distant group of stars.

* Visiting professor at Princeton University when this work was undertaken. The authors have shared evenly in the work; their names appear in alphabetical order. 
Very little work has been done on the origin of these motions. Von Weizsäcker $(1948,1949)$ has proposed that the motions can be regarded as turbulence generated by the differential galactic rotation, and he suggests that the spread of relative velocities within a region will vary as the cube root of the size of the region, as in isotropic turbulence. Such a picture has been applied by Jentzsch and Unsöld (1948) to an explanation of the equivalent widths of interstellar $N a$ I and $C a$ II. However, there is some question as to whether the theory of isotropic turbulence in an incompressible fluid can actually be applied to supersonic motions of a gas with large density fluctuations in a rotating galaxy.

Moreover, if we combine the evidence from interstellar lines with that of other observational data on dark clouds and luminous nebulae, the picture we obtain looks rather different from what would be expected on the theory of turbulent motion. Instead of more or less contiguous vortices, we find concentrated clouds that are often separated by much larger spaces of negligible density. Whenever two such clouds collide, there will be considerable losses of kinetic energy, and it is uncertain whether the transfer of energy from the rotation of the Galaxy, regarded as the largest eddy, to smaller and smaller eddies, as envisaged in the theory of isotropic turbulence, can maintain the velocities of the clouds at their observed level.

It would seem a priori that stellar radiation would, in principle, provide a much more abundant source of energy for the maintenance of cloud velocities. Spitzer and Savedoff (1950) have suggested that temperature differences between $H$ I and $H$ II regions might provide the driving force for the cloud motions, in much the same way as thermal effects produce terrestrial winds. No quantitative analysis along these lines was given.

It now seems probable that the major part of the kinetic energy of clouds may come from the formation of $\mathrm{O}$ stars. The fact that $\mathrm{O}$ stars are frequently found imbedded in, or associated with, large and dense cloud complexes indicates that they form preferentially in such extended nebulous regions. The newly born $\mathrm{O}$ star will ionize the hydrogen in its immediate surroundings up to the radius of the Strömgren sphere. In many cases the cloud agglomerations exceed this radius, and dense cool clouds remain at the outside, showing as dark nebulae. The ionization of the parts near the $\mathrm{O}$ star raises their temperature by a factor of 100 . The consequent increase of the pressure will cause the ionized parts of the cloud complex to expand and to push away the nonionized parts, which will, in general, be set moving with sensible velocities. These velocities will be increased by the circumstance that during the expansion the radius of the Strömgren sphere will increase faster than the radius of the expanding region and that, consequently, new parts of the neutral clouds will continually be ionized; the region of ionized $H$ will gradually eat its way into the $H$ I clouds.

A survey of various aspects of these phenomena has been given by Oort (1954), who discusses the observational material bearing upon the problem. Although the data are much too scant to permit a definite conclusion, it is indicated that the birth of $\mathrm{O}$ stars may well provide sufficient kinetic energy to maintain the random cloud motions and, besides, cause enough compression to explain the continued existence of compact interstellar clouds. It would also explain the phenomenon of the expansion of the associations of young stars.

To work out the actual details of what happens in a cloud complex subsequent to the birth of an $\mathrm{O}$ star would require much study. In the present article we wish to limit ourselves to one particular phase of the problem, namely, the calculation of the acceleration which the neutral part of a cloud undergoes as a consequence of the "evaporation" of neutral hydrogen by the radiation from the $\mathrm{O}$ star. The problem will be further simplified by assuming that the density in the region between the $\mathrm{O}$ star and the cloud is much lower than that of the cloud, so that the ionized layers can expand more or less freely in the direction toward the $\mathrm{O}$ star. The idealized case that we shall first consider is that 
JAN H. OORT AND LYMAN SPITZER, JR.

of an $\mathrm{O}$ star that has been formed at a certain distance from a dense cloud. It may be expected that the results obtained will, at least to some extent, be applicable to the more realistic case of $\mathrm{O}$ stars forming in a field of heavy, but irregularly distributed, clouds. We shall refer to this as case $a$. The second case $(b)$ which we propose to treat is that of a neutral cloud moving with a certain velocity into an $H \Pi$ region. In the third place, we shall consider the effects of the birth of $\mathrm{O}$ stars within a large, more or less homogeneous, mass of interstellar gas (case $c$ ). This last case, presumably the most realistic, is treated only very roughly here.

The general operation of the mechanism mentioned above is explained in Section II, while the detailed analysis is given in the following two sections. The final section gives some considerations relating to the distribution of high interstellar velocities.

\section{PROPOSED MECHANISM}

According to present theory, a typical cloud of neutral $H$ has a temperature in the general neighborhood of $100^{\circ} \mathrm{K}$ and a density of about $10 \mathrm{H}$ atoms $/ \mathrm{cm}^{3}$. This low temperature, predicted theoretically by Spitzer and Savedoff (1950), has since been confirmed by radio measures of the $H$ I emission line reported by Oort (1952), Wild (1952), and van de Hulst, Muller, and Oort (1954). An analysis by Strömgren (1948) shows that these assumed values of temperature and density are consistent with the observations of interstellar $\mathrm{Na}$ I and $\mathrm{Ca}$ II, provided that the composition of the interstellar gas is assumed to be about the same as in the stars. If the material between the clouds has a density of $0.1 \mathrm{H}$ atoms $/ \mathrm{cm}^{3}$, and a temperature of $10,000^{\circ} \mathrm{K}$, a cloud of density 10 and $T=100^{\circ}$ would be in pressure equilibrium with its surroundings.

Now consider what happens to a cloud when it becomes exposed to ultraviolet radiation from an $\mathrm{O}$ star. The $H$ atoms near the surface will evidently become ionized. The photoelectrons will escape with several volts of kinetic energy, and the kinetic temperature of the gas will rise, approaching some $10,000^{\circ}$ as the ionization approaches completeness. As the surface layer of gas becomes ionized, it will become transparent to ultraviolet radiation, and the region of ionized $H$ will eat its way into the $H$ I cloud.

The heating of the dense gas in the surface layer of the cloud will produce large dynamical effects. Evidently, an increase of temperature by a factor of 100 will increase the pressure by the same factor, and the gas will tend to expand vigorously. Expansion into the material of the cloud is soon stopped by the large amount of dense material there. In the direction away from the cloud, toward the O star, the heated gas "sees" only a low-density medium and expands in very much the same way as it would into a vacuum. Thus most of the cloud material ionized by the $O$ star will escape from the cloud. This material will be moving preponderantly in the direction of the $\mathrm{O}$ star, with a mean velocity relative to the cloud which we shall denote by $V$. Evidently, the escaping material must have an equal and opposite reaction on the cloud; in other words, it behaves precisely as a jet from a rocket and will accelerate the cloud away from the ionizing star.

In the simple one-dimensional case, the relation between the mass change and the velocity is the same as that used in all rocket computations. If $M$ is the mass of the cloud and $v$ the cloud velocity relative to the star, counted positive in the direction away from the star, then we have

$$
M \frac{d v}{d t}=-V \frac{d M}{d t}
$$

where the left-hand side is the rate of change of the cloud's momentum, while the righthand side is the momentum, relative to the cloud, carried away, per second, by the escaping gases. We have the usual solution

$$
M=M r e^{-\left(v-v_{0}\right) / V},
$$


where $v_{0}$ is the velocity at the time when the mass was $M_{0}$. In case $a$ we shall take $v_{0}=$ 0 ; in case $b$ we shall take it to be the velocity with which the cloud enters the $H$ II region.

In either of these two cases, it is evident from equation (2) that, to obtain velocity changes of the order of $V$ or larger, the mass of the cloud must decrease considerably. This is particularly true with case $b$; if the initial velocity of approach $v_{0}$ is small compared to $V$, it is readily shown that the final velocity is equal to $v_{0}$ but opposite in sign, and the collision is effectively elastic. If $v_{0}$ is comparable with $V$, the cloud mass $M$ is much greater during the initial deceleration than during the subsequent acceleration, and the cloud gains greater velocity than it loses; i.e., the collision will be superelastic in the sense that the cloud gains kinetic energy per unit mass by the encounter. ${ }^{1}$

\section{EJECTION OF GAS FROM A HEATED CLOUD}

We consider what happens to a relatively dense cloud of neutral $H$ when its surface becomes exposed to a flux $F_{u}$ ergs $\mathrm{cm}^{-2} \mathrm{sec}^{-1}$ of radiation beyond the Lyman limit. A detailed solution of this problem would evidently require a direct integration, with respect to time, of the hydrodynamic and thermal equations, since the problem can apparently not be idealized in terms of a steady-state solution. Evidently, a shock wave will advance into the neutral gas, the front of ultraviolet radiation will also move toward the rear of the cloud, while heated gas will stream out forward toward the star. No attempt at a detailed solution will be made here, and approximate estimates will be obtained for the quantities of interest.

We consider the amount of gas escaping per second and the mean velocity with which the gas escapes. If $M$ is the mass of the cloud, then $-d M / d t$ is the amount of material escaping per second. We may suppose that, on the average, $u$ ergs of ultraviolet energy are required for each $H$ atom (neutral or ionized) that escapes. Then, if $A$ is the projected area of the cloud, as seen from the central star,

$$
\frac{d M}{d t}=-\frac{A F_{u} m_{H}}{u}
$$

The presence of atoms other than $H$ may safely be ignored in this preliminary analysis.

To evaluate $d M / d t$, we must know $u$, the mean energy absorbed per escaping particle. The energy $u_{0}$ absorbed by a single $H$ atom, when it becomes photoionized, exceeds 13.5 ev by the kinetic energy of the ejected photoelectron. If the ionizing radiation is dilute black-body radiation with the color temperature $T_{c}$, the mean electron kinetic energy is nearly equal to $k T_{c}$, or about 2.5 ev near a star of surface temperature $30,000^{\circ}$ (Spitzer $1948 a$ ). Thus $u_{0}$ may be set equal to $16 \mathrm{ev}$, or $2.6 \times 10^{-11}$ ergs.

The ratio $u / u_{0}$, which we denote by $f$, equals the number of times an electron will be recaptured and reionized as the heated gas expands. This quantity is given by

$$
f=\overline{n \sigma_{p} v t}
$$

where $n$ is the electron density, equal, of course, to the proton density; $\sigma_{p}$ is the capture cross-section of a proton for an electron; and the bar denotes an average over all values of the electron velocity, $v$. The quantity $t$ is the time which an electron spends in the region of density $n$. It seems scarcely worth while to carry out the more refined computation, in which the variation of $n$ along the electron path is considered.

The effective recombination coefficient, $\overline{\sigma_{p} v}$, for capture of electrons by protons has been computed by Spitzer (1948a) and others, and may be written

$$
\overline{\sigma_{p} v}=2 A\left(\frac{2 k T}{\pi m_{e}}\right)^{1 / 2} \beta \phi(\beta),
$$

${ }^{1}$ As pointed out to us by Dr. Burgers, it may be shown generally that, because of the decrease of mass, the total kinetic energy of the cloud, measured relative to the $O$ star, always decreases. Relative to the local standard of rest, however, the energies of the clouds may be increased by these encounters. 
where $A$ is $2.11 \times 10^{-22} \mathrm{~cm}^{2}, \beta$ equals $158,000 / T$, and $\phi(\beta)$ is given in Table 1 of the paper by Spitzer $(1948 a)$. If $T$ is $10,000^{\circ}$, we find

$$
\overline{\sigma_{p} v}=4.5 \times 10^{-13} \mathrm{~cm}^{3} / \mathrm{sec} .
$$

Thus a typical electron will recombine in a time equal to $\left(n \overline{\sigma_{p} v}\right)^{-1}$, or $7 \times 10^{4} / n$ years.

The factors $n$ and $t$ entering into the expression for $f$ may be estimated from the following simple argument. Suppose a dense cloud to become exposed to radiation from an $\mathrm{O}$ star that has been formed in its vicinity. The radiation will at first ionize the cloud at a rapid rate, causing a stream of ionized particles to proceed from the cloud. As the stream develops, it will absorb more and more of the ultraviolet radiation coming from the $\mathrm{O}$ star, thereby diminishing the amount available for ionization of the cool cloud. The stream will form an insulating shell around the cool cloud. A semistationary state may be reached when the density in the stream has become such that the intensity of the radiation penetrating to the surface of the cool cloud is just sufficient to maintain the stream.

To deal properly with the problem of this "insulating" layer would seem to require an extensive investigation, which we have not yet been able to carry out. We realize that it introduces a serious uncertainty in some of the results derived. Since our analysis was carried out, Dr. Kahn (1954a) has investigated the expansional and compressional motions that will be set up in a plane, cool cloud as a consequence of the heating of its surface by the radiation of an $\mathrm{O}$ star. In the present investigation we have confined ourselves to a rather simple estimate of the total outward momentum which will be imparted to the part of the cloud that remains un-ionized, as a consequence of the evaporation of particles from the surface exposed to the radiation of the $\mathrm{O}$ star.

To obtain a rough estimate of the influence of the insulating shell, we assume that the stream of ionized matter proceeds relative to the cloud with a velocity of the order of $V$, for which we shall take $20 \mathrm{~km} / \mathrm{sec}$. The amount of material in the shell may be computed in the following manner. If the outward velocity of the material in the shell is constant and directed radially outward from the cloud center, then the equation of continuity requires that the density of matter vary as $1 / R^{2}$, where $R$ is the distance from the center of the cloud. The total amount of material per square centimeter in the line of sight between the cloud surface and the star is then equal to the density at the cloud surface multiplied by the "effective thickness," which in this case is the cloud radius, $R_{c}$. If the absorption coefficient per gram varies as some power of the density, the effective thickness will be reduced below $R_{c}$, and we shall here take an effective thickness equal to $\frac{1}{2} R_{c}$.

We now consider a cloud of radius $R_{c}$ subjected to the radiation of an $\mathrm{O}$ star at a distance $r$. Suppose that without absorption this radiation were capable of ionizing a mass $g / r^{2} \mathrm{~cm}^{-2} \mathrm{sec}^{-1}$. Suppose, further, that the radiation is reduced by a factor $f$ through absorption in the ionized shell. The number of protons and electrons developed per square centimeter per second is then $g /\left(f m_{H} r^{2}\right)$. The number $n$ of protons per cubic centimeter of the stream is

$$
n=\frac{g}{f m V r^{2}} \text {. }
$$

With a thickness of $R_{c} / 2$ for the shell, the expansion through this thickness will require that

$$
t=\frac{R_{c}}{2 V}
$$

Per electron, the number of recombinations during its passage through the shell (which 
number is at the same time the factor by which the radiation effective for the ionization is reduced) will be, on substitution of equations (6), (7), and (8) in equation (4),

$$
f=\frac{2.3 \times 10^{-13} g R_{c}}{f m_{H} V^{2} r^{2}}
$$

Hence

$$
f=\frac{1}{V r}\left(\frac{2.3 \times 10^{-13} g R_{c}}{m_{H}}\right)^{1 / 2}=0.18 \frac{\left(g R_{c}\right)^{1 / 2}}{r} .
$$

Values of $g$ have been tabulated in Table 1, appearing in the next section. With $R_{c}=$ $5 \mathrm{pc}, r=10 \mathrm{pc}$, we obtain for an 05 star, $f=66$; for O7, $f=28$; and for O9, $f=9$. For larger $r, f$ diminishes proportional to $r$. Considering, similarly, a small dense cloud, with $R_{c}=1 \mathrm{pc}$, we obtain, at $r=2 \mathrm{pc}$, values of $f$ equal to 147,63 , and 22 for stars of types $05, \mathrm{O} 7$, and $\mathrm{O} 9$, respectively. We may also express these results by saying that the effective value of $u$ should be taken larger than $2.6 \times 10^{-11}$ by the factors mentioned.

In the case of an 05 star the density in the insulating shell would become 40 for the first cloud and 452 for the second. Considering that the shell is formed only on that half of the cloud's surface that receives radiation from the $O$ star, the volume of the shell is 1.2 times that of the original cloud. This means that, in the two examples considered, the original density of the cloud should be at least 50 and $550 \mathrm{H} / \mathrm{cm}^{3}$ in order not to be vaporized entirely before the insulating shell has been formed. For O stars of later type the minimum densities are smaller.

We turn to a consideration of the mean ejection velocity. The gas may be assumed to leave the cloud at a velocity $V_{e}$ normal to the cloud's surface; the mean component of this velocity in the direction of the central star will be $V_{e} \cos \theta$ where $\theta$ is the angle which the direction to the star makes with the outward normal. Evidently, $V_{e}$ will depend on the temperature to which the ionized gas is heated. Two situations will be considered-one in which the photoelectrons are not recaptured by protons before the gas escapes; the other, in which they are recaptured and re-emitted a number of times.

In the first of these situations the temperature will depend entirely on the initial kinetic energy of the photoelectrons. The mean energy of a photoelectron from $H$ is equal to $k T_{c p}$, where $T_{c p}$ is roughly the color temperature of the ionizing radiation (Spitzer $1948 a$ ). In this case we may assume that virtually all this electron energy goes into the expansion velocity, with electrons and protons accelerated equally, and write

$$
V_{e}^{2}=\frac{2 k T_{c p}}{m_{H}}
$$

Values of $V_{e}$ obtained from this equation range from $19 \mathrm{~km} / \mathrm{sec}$ for a star of type B0 to $26 \mathrm{~km} / \mathrm{sec}$ for type $\mathrm{O} 7$ and up to $31 \mathrm{~km} / \mathrm{sec}$ for type 05 .

It is readily shown that if electron captures by protons are negligible, excitation of forbidden lines by electron impact will also have a negligible effect. However, the possible presence of $\mathrm{H}_{2}$ molecules must be considered, since, as shown by Spitzer (1949), the rate of radiation by $\mathrm{H}_{2}$ molecules is very rapid when the temperature is high. Because of the high cosmic abundance of $H$, encounters between electrons and $H_{2}$ molecules may dissipate appreciable energy, even though there is not sufficient time for electrons to lose energy in collisions with oxygen and other atoms.

While radiation by $H_{2}$ molecules may be appreciable, it appears possible that this loss of energy may be offset by the corresponding energy gain resulting from dissociative recombination,

$$
H_{2}^{+}+e \rightarrow 2 H
$$


As pointed out by Bates (1950), this process may have a very high rate coefficient (greater by a factor of some $10^{5}$ than the rate coefficient for electron capture by protons). If the two $H$ atoms are both in their ground states, each atom will have a kinetic energy of 5.5 volts, plus half the kinetic energy of the incident electron. Some of this energy will be radiated in impacts with other $H_{2}$ or $H_{2}^{+}$molecules, and it is not clear whether, on the whole, the presence of molecules will increase or decrease $V_{e}$. For preliminary results we shall assume that equation (11) is valid even if some $H_{2}$ molecules are present.

When the electrons recombine with protons and absorb ultraviolet photons several times in the course of the expansion, we may assume that the temperature is determined not solely by the initial electron energy but rather by the condition of equilibrium between the gains and losses of kinetic energy. The kinetic temperature is then about the same as in planetary nebulae and $H$ II regions generally; we may take $10,000^{\circ}$ as a representative value. In this situation the temperature tends to remain constant as the gas expands.

The final expansion velocity may be computed very simply. As a gram of matter expands, it does work equal to $p d(1 / \rho)$. If we assume that this work goes entirely into increasing the expansion velocity of this particular gram, then we find

$$
V^{2}+\frac{2 k T}{m} \ln \rho=\text { Constant. }
$$

If we let the original density be $\rho_{0}$, the final density be $\rho_{1}$, and again let $m$, the mean mass per particle, equal $m_{H} / 2$, then

$$
V_{e}^{2}=\frac{4 k T}{m_{H}} \ln \left(\frac{\rho_{0}}{\rho_{1}}\right)
$$

If $\rho_{0}$ and $\rho_{1}$ correspond to particle densities of 10 and $0.1 H$ atoms $/ \mathrm{cm}^{3}$, typical values for $H$ I clouds and for the regions between the clouds (Strömgren 1948), we find that equation (13) gives a value of $39 \mathrm{~km} / \mathrm{sec}$ for $V_{e}$, if $T$ is set equal to $10^{4}$.

It may be remarked that in this case, where the expansion is assumed to occur relatively slowly compared to the ionization, the presence of $H_{2}$ molecules may be neglected. Any molecules present will be dissociated relatively early and can have no effect on the temperature during most of the gradual expansion.

Finally, we must average $V$, or $V_{e} \cos \theta$ over a hemisphere. If we assume that the loss of mass per unit cloud surface is proportional to the projected area, or to $\cos \theta$, we must average $\cos \theta$ over $\cos \theta d \omega$, where $d \omega$ is an element of solid angle. In this way we obtain, for a spherical cloud,

$$
V=\frac{2}{3} V_{e} .
$$

While interstellar clouds are probably not even remotely spherical, equation (14) should provide a fair approximation, on the average. In conclusion, we have the following range of values for $V$,

$$
13 \leq V \leq 26 \mathrm{~km} / \mathrm{sec} .
$$

A value of $20 \mathrm{~km} / \mathrm{sec}$ for $V$ has been adopted throughout most of this paper.

\section{DYNAMICS OF CLOUDS ACCELERATED BY RADIATION}

If the gas in the $H$ II region is assumed initially homogeneous, the ultraviolet flux depends on the distance, $r$, from the central star, according to the equation

$$
F_{u}=\frac{L_{u}}{4 \pi r^{2}}\left(1-\frac{r^{3}}{s_{0}^{3}}\right) \quad\left(r<s_{0}\right)
$$


where $L_{u}$, the luminosity of the star beyond the Lyman limit, is related to the stellar radius $R$ and the surface temperature $T_{c}$ by the approximate relationship

$$
L_{u}=4 \pi R^{2} \times \frac{2 \pi h}{c^{2}} \int_{\nu_{0}}^{\infty} \nu^{3} e^{-h \nu / k T_{c}} d \nu
$$

where $\nu_{0}$ is the frequency at the Lyman limit. In the following discussion we shall, in general, suppose the medium to have a density of 1 proton and electron per cubic centimeter. We define $s_{0}$ as the radius of the Strömgren sphere in such a medium. Equation (16), together with values of $s_{0}$ for stars of different spectral type, may be taken from Strömgren (1939). Evidently $F_{u}$ vanishes for $r$ greater than $s_{0}$. If equations (3), (16), and (17) are combined, we may write the resultant equation in the form

$$
\frac{d M}{d t}=-\frac{\pi g R_{c}^{2}}{f r^{2}}\left(1-\frac{r^{3}}{s_{0}^{3}}\right)
$$

where $M$ and $R_{c}$ are the cloud's mass and radius. The constant $g$ is given by

$$
\begin{aligned}
g=\frac{m_{H} L_{u}}{4 \pi u_{0}} & =\frac{2 \pi R^{2} m_{H} h}{u_{0} c^{2}} \int_{\nu_{0}}^{\infty} \nu^{3} e^{-h \nu / k T_{c}} d \nu \\
& =\frac{2 \pi R^{2} m_{H} k T_{c} \nu_{0}^{3} e^{-\beta}}{u_{0} c^{2}}\left(1+\frac{3}{\beta}+\frac{6}{\beta^{2}}+\frac{6}{\beta^{3}}\right),
\end{aligned}
$$

where

$$
\beta=\frac{h \nu_{0}}{k T_{c}}=1.58 \times 10^{5} T_{c}^{-1} .
$$

Again $f$ is the factor by which the radiation is reduced by absorption in the stream of ionized particles developing from the cloud's surface. In the case of an isolated cloud we shall use equation (10) for $f$. Equation (18) may then be written

$$
\frac{d M}{d t}=-5.5 \pi\left(g R_{c}^{3}\right){ }^{1 / 2} \frac{1}{r}\left(1-\frac{r^{3}}{s_{0}^{3}}\right)
$$

It should be noted that, in the computation of $f$ from equation (10), the effect of the reduction of the radiation by the general interstellar medium has been neglected. This medium gives an additional factor $\left(1-r^{3} / s_{0}^{3}\right)^{1 / 2}$ in $f$, and strictly, therefore, in equation (21) the factor $\left(1-r^{3} / s_{0}^{3}\right)$ should be replaced by $\left(1-r^{3} / s_{0}^{3}\right)^{1 / 2}$. In reality, however, the phenomena will be more complicated, because the insulating layer will dissipate only gradually after the radiation has been cut off. We have therefore left the factor as in equation (21). The change to a square root would not have produced a very great change in the results.

Values of $g$ computed from equation (19) are given in Table 1, for stars of different spectral type. The values of $T_{c}$ and $R$ are practically those used by Strömgren (1939); the values of $s_{0}$, also from Strömgren, are for a gas density of one $H$ atom $/ \mathrm{cm}^{3}$. The value of $V$ is set equal to $20 \mathrm{~km} / \mathrm{sec}$, while $u_{0}$ has been set equal to $3 \times 10^{-11}$ ergs.

We shall now consider three different cases of acceleration of interstellar clouds:

a) An $\mathrm{O}$ star is formed at a distance $r_{0}$ from an isolated cloud, where $r_{0}$ is smaller than the radius of the Strömgren sphere in the average interstellar medium (supposed to have a density of 1 proton and electron per cubic centimeter). It will be assumed that the $\mathrm{O}$ star suddenly begins to radiate at full strength. This supposition simplifies the analysis but is not essential for the theory. 
b) An isolated cloud moves into an $H$ II region.

c) An $\mathrm{O}$ star, or a group of $\mathrm{O}$ stars, is formed inside a homogeneous cloud of large dimension and considerable density.

CASE $a$. REPULSION OF AN ISOLATED CLOUD BY A NEWLY FORMED O STAR

We shall first consider the case where, in the space between the $\mathrm{O}$ star and the cloud, the influence of the general medium is negligible. If we assume, for simplicity, that the cloud radius $R_{c}$ is constant during the acceleration and combine equations (1), (2), and (21), we find

$$
\frac{d v}{d t}=-\frac{V}{M} \frac{d M}{d t}=\frac{5.5 \pi V\left(g R_{c}^{3}\right)^{1 / 2}}{M_{0} r} e^{\left(v-v_{0}\right) / v} .
$$

If we substitute $v d / d r$ for $d / d t$, equation (22) can be integrated directly, to give

$$
(v+V) e^{-\left(v-v_{0}\right) / v}=-17.2 \frac{\left(g R_{c}^{3}\right) 1 / 2}{M_{0}} \ln \left(\frac{r}{r_{0}}\right)+v_{0}+V,
$$

where $\ln$ is the natural logarithm.

TABLE 1

VALUES OF $g$ AND $\gamma$

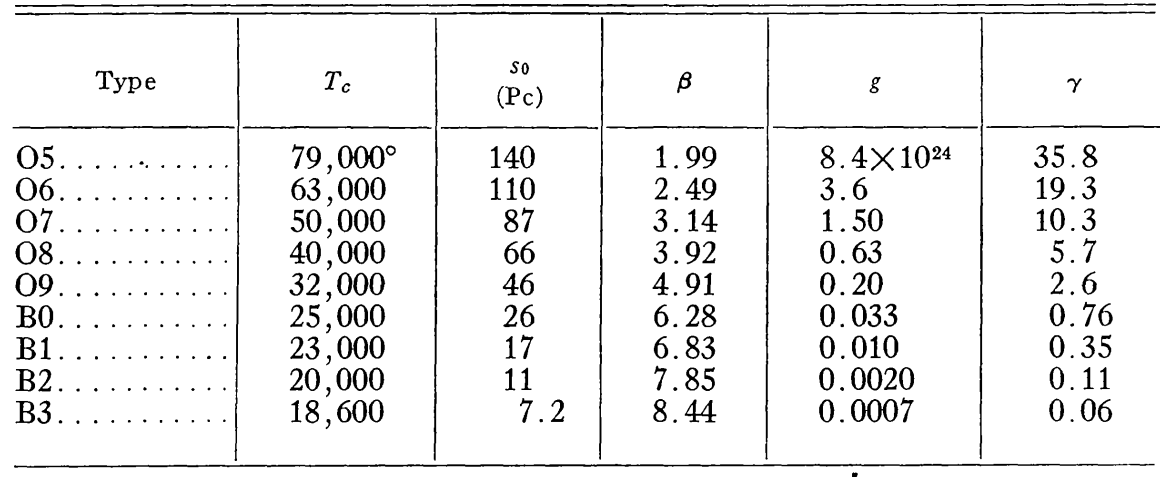

So far, the effect of the reduction of the radiation by the general interstellar medium has been neglected. Should we assume that, in addition to the protective layer surrounding the cloud, there is an ionized medium of density 1 hydrogen particle $/ \mathrm{cm}^{3}$, the ultraviolet radiation would be cut off at $r=s_{0}$. Integration of equations (1), (2), and (21) then gives, for $r=s_{0}$,

$$
(v+V) e^{-\left(v-v_{0}\right) / V}=-17.2 \frac{\left(g R_{c}^{3}\right)^{1 / 2}}{M_{0}}\left\{\ln \left(\frac{s_{0}}{r_{0}}\right)-\frac{1}{3}\left(1-\frac{r_{0}^{3}}{s_{0}^{3}}\right)\right\}+v_{0}+V .
$$

In most relevant cases $s_{0} / r_{0}$ will be larger than 10 , and the second term within the braces will be small compared to the first. In order to compute the final velocities obtained, we may therefore safely use equation (23), putting $r=s_{0}$.

Even if the general ionized medium between the cloud and the star should remain negligible, there will be a limit to the distance up to which the cloud is accelerated. This limit will be set by the finite lifetime of the $O$ star. If for the brightest $O$ stars we put this at 5 million years and if we suppose the average velocity of the cloud to be $30 \mathrm{~km} / \mathrm{sec}$, the cloud travels to a distance of $150 \mathrm{pc}$ before the $\mathrm{O}$ star declines in brightness.

A third factor which may limit the acceleration of the cloud is the sweeping-up of "ordinary" interstellar material. According to equation (7), the number of protons and 
electrons evaporated per second from $1 \mathrm{~cm}^{2}$ of the cloud, if there is no general medium between the cloud and the $O$ star, is $g /\left(f m_{H} r^{2}\right)$, or $5.6 \mathrm{~g}^{1 / 2} /\left(r m_{H} R_{c}^{1 / 2}\right)$. If the medium in front of the cloud has a density of 1 atom $/ \mathrm{cm}^{3}$, the number of atoms swept up at the front side is $v \mathrm{~cm}^{-2} \mathrm{sec}^{-1}$. Each evaporating proton carries away a momentum $m_{H} V$, while each atom swept up adds a momentum $m_{H} v$ in the same direction. The two amounts of momentum will be equal when $5.6 g^{1 / 2} V /\left(r R_{c}^{1 / 2}\right)=m_{H} v^{2}$. For an O5 star and a cloud of 5-pc radius the braking action will become of the same order as the acceleration at $r=180 \mathrm{pc}$ if $v=30 \mathrm{~km} / \mathrm{sec}$, and at $410 \mathrm{pc}$ if $v=20 \mathrm{~km} / \mathrm{sec}$. This effect may therefore be of the same order as the first two effects.

From equation (23) we can derive the limiting original mass that a cloud must have in order to escape total ionization. This limiting mass, $M_{0}$, is obtained by putting the right-hand member equal to zero. As an example, we may consider the case of a cloud of 5-pc radius accelerated from $r_{0}=10$ to $r=100 \mathrm{pc}$. If $v_{0}=0$ and $V=20 \mathrm{~km} / \mathrm{sec}$, we find, from equation (23) and Table 1 , that $M_{0}=1700$ solar masses for an 05 star, and 700 solar masses for an $\mathrm{O} 7$ star.

Clouds with original masses slightly exceeding these values will attain high velocities. It is tempting to think that the high interstellar velocities observed in some cases are due to this rocket effect. It may be seen from equation (23) that there is a considerable range of $M_{0}$ where the accelerations will be large. Acceleration to rather high velocities will therefore occur not infrequently.

One of the most interesting cases observed is that of the California nebula NGC 1499. Mayall (1953) has recently measured a radial velocity of $+53 \mathrm{~km} / \mathrm{sec}$ for this nebula; the velocity is rather uncertain, and Mayall thinks that it is likely to be the same as that of the exciting $O$ star $\xi$ Persei, which has a radial velocity of $+67 \mathrm{~km} / \mathrm{sec}$. It is probable that, notwithstanding its large velocity of $49 \mathrm{~km} / \mathrm{sec}$ with respect to the center of the $\zeta$ Persei association, $\xi$ Persei belongs to this group (Blaauw 1952a; Delhaye and Blaauw 1953), in which case NGC 1499 should also belong to it. If we think of NGC 1499 as having been expelled from the center of the group by the ionization effects of an early $\mathrm{O}$ star, it is natural to suppose that $\xi$ Persei likewise obtained its high velocity in this manner. It should then have been formed in the cloud after this had obtained most of its acceleration, and probably as a consequence of the compression accompanying this acceleration (Oort 1954). At present, the $\zeta$ Persei association contains no $O$ stars besides $\xi$ Persei itself. If the foregoing supposition concerning the origin of the high velocities of NGC 1499 and $\xi$ Persei is correct, there should originally have been an $\mathrm{O}$ star, which must have faded during the 1.5 million years elapsed since the origin of the association.

The dimensions of NGC 1499 are roughly $13 \times 4 \mathrm{pc}$. A thickness of $10 \mathrm{pc}$ in the radial direction and a density of $20 \mathrm{H} / \mathrm{cm}^{3}$ might be reasonable estimates, in view of the observed brightness. With these estimates, the mass of the luminous nebula would be 130 solar masses. The total mass may be larger, as not all of the cloud may be ionized. For the present we shall assume a mass equal to 130 times that of the sun. Equation (2) then enables us to determine $M_{0}$. With $v_{0}=0, v=49 \mathrm{~km} / \mathrm{sec}, V=20 \mathrm{~km} / \mathrm{sec}$, we find $M / M_{0}=0.086$, or $M_{0}=1500$ solar masses.

As we shall see in the discussion of case $c$, it may well be that the clouds, before they become isolated, have already obtained a velocity $v_{0}$ of the order of the velocity of sound in an $B$ II region, or about $11 \mathrm{~km} / \mathrm{sec}$. Also, $V$ may, in reality, be as high as $30 \mathrm{~km} / \mathrm{sec}$. With these values for $v_{0}$ and $V$, we would get

$$
\frac{M}{M_{0}}=0.28, \quad \text { or } \quad M_{0}=460 \text { solar masses . }
$$

Supposing that the cloud was accelerated without expansion from $r_{0}=8$ to $r=50 \mathrm{pc}$, we can now derive, from equation (23), the value of $g$ that would have been needed to accelerate the cloud to a velocity $v=49 \mathrm{~km} / \mathrm{sec}$. Taking $R_{c}=4 \mathrm{pc}$, we find, for the 
JAN H. OORT AND LYMAN SPITZER, JR.

two cases considered, $g=9.8 \times 10^{24}$ and $g=1.6 \times 10^{24}$, respectively. From Table 1 we see that the first value corresponds to a star of type O5, while in the second case a star of type $\mathrm{O} 7$ would have sufficed. With an 05 star at 8-pc distance, the density in the insulating layer, by equations (10) and (7), would be roughly $60 \mathrm{H} / \mathrm{cm}^{3}$. As the density in the original cloud would in this case be $20 / 0.086=233 \mathrm{~cm}^{-3}$, the mass of the insulating shell would be about one-third that of the cloud. The velocity imparted to the cloud as a consequence of the formation of the insulating shell has been neglected in the foregoing estimates. If we had taken it into account, we would have found that a star of slightly lower temperature would have sufficed to give the cloud the required velocity. With somewhat different assumptions, as, for example, the assumption that $R_{c}$ changes as the cloud accelerates outward, the computed value of $g$ will be altered by a factor of 2 or more.

Strictly, this analysis applies to a neutral cloud, while what we see of NGC 1499 is an ionized mass. The picture would be that the nebula has been accelerated by the rocket effect while it was still in the neutral state. Evidently, $\xi$ Persei was formed after the acceleration was practically finished, for it has the same velocity as the nebula. It is this star which afterward ionized the cloud.

A much more extreme case of high velocity is presented by the $O$ star AE Aurigae. The unusual motion of this star has been discussed by Blaauw and Morgan (1953), who point to the possibility that the star originated in the Orion association. The star has a space velocity of $128 \mathrm{~km} / \mathrm{sec}$ (corrected for standard solar motion) that appears to be pointed away from the Orion group; it is situated at a distance of $350 \mathrm{pc}$ from the center of this group. Like $\xi$ Persei, it appears to be associated with a rather dense, bright nebula, IC 405. It is not unlikely that this nebula moves with the star. The radius of the cloud is estimated by Blaauw and Morgan to be $2.8 \mathrm{pc}$, its density $50 \mathrm{H} / \mathrm{cm}^{3}$, which would correspond to a total mass of 80 times that of the sun. We again make two estimates of the original mass, $M_{0}$. If we take $v_{0}=0, V=20 \mathrm{~km} / \mathrm{sec}$, the mass reduction factor needed to reach a velocity of $128 \mathrm{~km} / \mathrm{sec}$ is $M / M_{0}=0.0017$, so that $M_{0}=48,000$ solar masses. If, on the other hand, we assume $v_{0}=11 \mathrm{~km} / \mathrm{sec}, V=30 \mathrm{~km} / \mathrm{sec}$, we get $M / M_{0}=0.020$, and $M_{0}=4000$ solar masses. The first value is improbably high. It seems that if the velocity of $\mathrm{AE}$ Aurigae were to be explained by the rocket mechanism, $V$ would have to be at least $30 \mathrm{~km} / \mathrm{sec}$. Entering equation (23) with this value, with $v_{0}=11 \mathrm{~km} / \mathrm{sec}, v=128 \mathrm{~km} / \mathrm{sec}, M_{0}=4000$ solar masses, $R_{c}=2.8 \mathrm{pc}, r_{0}=2 R_{c}=$ $5.6 \mathrm{pc}$, and $r=350 \mathrm{pc}$, we find $g=280 \times 10^{24}$. Comparing this with the values given in Table 1, we see that it would require 34 O5 stars to give to a cloud of 80 solar masses a velocity of $128 \mathrm{~km} / \mathrm{sec}$. Such a collection of early $\mathrm{O}$ stars appears very improbable.

The exceptional character of this case has been shown to be still more remarkable by Blaauw and Morgan's (1954) determination of the motion of $\mu$ Columbae. They point out that the B0 V star $\mu$ Columbae moves away from the Orion association with the same speed as AE Aurigae and that the two motions are almost exactly opposite. They suggest that the stars were formed in the same physical process and that this took place in the Orion nebula. This phenomenon indicates that the mechanism of the formation of these two high-velocity stars has been radically different from the processes considered in the present article.

In most other cases of high-velocity $\mathrm{O}$ stars or of high-velocity clouds found from interstellar absorption lines, the velocities lie between 50 and $75 \mathrm{~km} / \mathrm{sec}$; i.e., in a range where they can be explained without difficulty by rocket effects.

\section{CASE $b$. ENCOUNTER OF A CLOUD WITH AN $H$ II REGION}

Because case $b$ is probably less important for the dynamics of interstellar space than cases $a$ and $c$, we shall slightly simplify the discussion by limiting ourselves to a consideration of "standard" clouds and "standard" $H$ II regions. Following Strömgren (1948), we assume a "typical" cloud to have a thickness, $d$, of 5 parsecs and a gas density, $n_{H}$, of 
$10 H$ atoms $/ \mathrm{cm}^{3}$. If we call $n_{H} d$ the mass thickness, this mass thickness is therefore 50 , or $2.7 \times 10^{-4} \mathrm{gm} / \mathrm{cm}^{2}$.

We may now define a dimensionless quantity, $\gamma$, as follows:

$$
\gamma=\frac{g A_{0}}{V M_{0} s_{0}}
$$

in which $A_{0}$ and $M_{0}$ are the cloud's original projected area and mass, which were assumed to be those of the "typical" cloud discussed above. Values of $\gamma$ have been given in Table 1. The various constants involved were given the same values as for the computation of $g$. As we shall see later, encounters between a cloud and an $H$ II region are relatively ineffective in accelerating clouds to high velocities, unless $\gamma$ exceeds unity. Evidently, the early B stars yield so low a value of $\gamma$ that they may safely be neglected.

In the present computation we shall set $f$ equal to unity and neglect the formation of the insulating layer. Mechanism $b$ is important only when $\gamma$ is larger than unity, and in such a case the cloud does not come very far inside the Strömgren sphere. The evaporation rate then remains relatively low, and $f$ will, in fact, not much exceed unity.

Equation (18) may now be written

$$
\frac{d M}{d t}=-\frac{\gamma s_{0} V M_{0}}{r^{2}}\left(1-\frac{r^{3}}{s_{0}^{3}}\right) \frac{A}{A_{0}},
$$

$A$ being the area at the time $t$ which is considered. As before, we shall assume that $A$ remains constant during the acceleration.

While the general problem is, of course, two-dimensional, we shall gain adequate insight into the physical situation by considering the simple one-dimensional case of a direct central collision. In this case $v$ is $d r / d t$, equation (2) is applicable, and, if equations (1), (2), and (26) are combined, we obtain, for $r<s_{0}$

$$
\frac{d v}{d t}=-\frac{V}{M} \frac{d M}{d t}=\gamma s_{0} V^{2} e^{\left(v-v_{0}\right) / V} \frac{1}{r^{2}}\left(1-\frac{r^{3}}{s_{0}^{3}}\right) .
$$

Integration with respect to $r$ gives

$$
\left(1+\frac{v}{V}\right) e^{-\left(v-v_{0}\right) / V}-\left(1+\frac{v_{0}}{V}\right)=\gamma\left\{\frac{s_{0}}{r}+\frac{1}{2}\left(\frac{r}{s_{0}}\right)^{2}-\frac{3}{2}\right\},
$$

where use has been made of the boundary condition that $v=v_{0}$, the initial velocity of approach, when $r=s_{0}$. The velocity is taken positive when directed away from the star; thus $v_{0}$ is negative.

At the boundary of the $H$ II region the right-hand side of equation (28) vanishes; if we denote by $v_{1}$ the velocity of the outgoing cloud when $r=s_{0}$ again, it is evident that $v_{1}$ must be such that the left-hand side of equation (28) vanishes for $v=v_{1}$. It is readily seen that if $\left|v_{0}\right|>V$, the left-hand side vanishes only for the one value $v=v_{0}$. This corresponds to the physical fact that, for such large velocities of approach, the cloud loses all its mass before it escapes from the $H$ II region. For $v_{0}$ negative and with an absolute value less than $V$, a positive value of $v_{1}$ can be found for which this left-hand side vanishes. Values of $v_{1} / V$ for different $v_{0} / V$ are given in Table 2. For small $v_{0} / V$ we have

$$
\frac{v_{1}}{V}=\frac{\left|v_{0}\right|}{V}+\frac{2}{3}\left(\frac{v_{0}}{V}\right)^{2} \ldots
$$

while, for $\left|v_{0}\right| / V$ very near unity,

$$
\frac{v_{1}}{V}=\ln \frac{1}{1+v_{0} / V}
$$


Equation (28) also indicates how close to the central star the cloud comes before its velocity vanishes. If we denote this distance of closest approach by $r_{m}$, we have

where

$$
\frac{r_{m} / s_{0}}{1+0.5\left(r_{m} / s_{0}\right)^{3}}=\frac{1}{1.5+0.5 \Psi\left(v_{0} / V\right)}
$$

$$
\Psi(y)=e^{y}-(1+y) \text {. }
$$

For small $v_{0} / V, \Psi$ varies as $\frac{1}{2}\left(v_{0} / V\right)^{2}$, but increases to 0.368 for $v_{0}$ equal to $-V$. The values of $r_{m} / s_{0}$ found from equation (31) for different values of $\gamma$ and $\left|v_{0}\right| / V$ are given in Table 3. Evidently, for $\gamma$ large compared to unity, the right-hand side of equation

TABLE 2

EMERgent VeLocity For DiRECT CENTRAL ENCOUNTER

\begin{tabular}{l|l|l|l|l|l|l|l|l|l|l}
\hline \hline$-v_{0} / V \ldots \ldots \ldots \ldots$ & 0.10 & 0.20 & 0.30 & 0.40 & 0.50 & 0.60 & 0.65 & 0.70 & 0.75 & 0.80 \\
$v_{1} / V \ldots \ldots \ldots \ldots$ & 0.107 & 0.231 & 0.376 & 0.547 & 0.756 & 1.02 & 1.18 & 1.36 & 1.59 & 1.86 \\
$-v_{0} / V \ldots \ldots \ldots \ldots$ & 0.82 & 0.84 & 0.86 & 0.88 & 0.90 & 0.92 & 0.94 & 0.96 & 0.98 & 0.99 \\
$v_{1} / V \ldots \ldots \ldots \ldots$ & 1.99 & 2.14 & 2.30 & 2.49 & 2.72 & 2.99 & 3.34 & 3.83 & 4.67 & 5.48 \\
\hline
\end{tabular}

TABLE 3

\begin{tabular}{|c|c|c|c|c|c|c|c|}
\hline \multirow{2}{*}{$\gamma$} & \multicolumn{7}{|c|}{$-v_{0} / V$} \\
\hline & 0.1 & 0.2 & 0.3 & 0.4 & 0.6 & 0.8 & 1.0 \\
\hline $\begin{array}{c}0.1 \ldots \\
0.3 \ldots \\
1.0 \ldots \\
3 \ldots \ldots \\
10 \ldots \ldots\end{array}$ & $\begin{array}{r}0.831 \\
.900 \\
.944 \\
.968 \\
0.982\end{array}$ & $\begin{array}{r}0.690 \\
.810 \\
.893 \\
.937 \\
0.965\end{array}$ & $\begin{array}{r}0.574 \\
.730 \\
.844 \\
.908 \\
0.949\end{array}$ & $\begin{array}{r}0.479 \\
.659 \\
.800 \\
.880 \\
0.933\end{array}$ & $\begin{array}{r}0.341 \\
.541 \\
.719 \\
.830 \\
0.904\end{array}$ & $\begin{array}{r}0.252 \\
.435 \\
.650 \\
.784 \\
0.877\end{array}$ & $\begin{array}{r}0.194 \\
.368 \\
.591 \\
.742 \\
0.852\end{array}$ \\
\hline
\end{tabular}

VALUE OF $r_{m} / s_{0}$ FOR CENTRAL ENCOUNTERS

(31) is nearly equal to $1 / 1.5$, and $r_{m}$ is only slightly less than $s_{0}$; in this case one may expect equation (28) to be valid for noncentral encounters. For $\gamma$ less than unity and $v_{0}$ comparable with $-V, r_{m}$ is much less than $s_{0}$; these results are applicable only to the improbably few direct central encounters.

While this mechanism $(b)$ can account qualitatively for the existence of high-velocity clouds, the number of such clouds appears to be greater by about an order of magnitude than would be expected from the mechanism. The number of $\mathrm{O}$ stars per cubic parsec in the solar neighborhood ranges from about $2 \times 10^{-9}$ at $\mathrm{O} 5$ to $1.6 \times 10^{-8}$ at $\mathrm{O} 9$. The radius of the $H$ II sphere around each star may be taken from Table 1 . With conventional values for the cloud velocity, for the number of clouds per kiloparsec, and for the mean free path of a cloud, it is readily shown that the number of high-velocity clouds produced by mechanism $b$ is smaller by a factor of 20 than the number computed from the observations by Adams.

\section{CASE $c$. BIRTH OF O STARS INSIDE A LARGE CLOUD}

Interstellar clouds sometimes seem to collect together and to form a conglomerate, with a mass that is vastly greater than that of a "typical" cloud. We thus find cloud 
complexes with masses of several ten thousand times the mass of the sun. Apparently, $O$ stars can be formed in such cloud complexes, for we observe several instances where $\mathrm{O}$ stars are imbedded in dense clouds of very large dimensions.

We now wish to consider what happens when an $\mathrm{O}$ star is formed in such a dense medium. We shall first take an idealized case, where an $\mathrm{O}$ star is suddenly born in the central part of a vast, homogeneous, spherical cloud with a density of $n_{0}$ hydrogen atoms $/ \mathrm{cm}^{3}$. As before, let $s_{0}$ denote the radius that the Strömgren sphere around the $\mathrm{O}$ star would have if it were situated in a medium with a density of 1 hydrogen atom/ $\mathrm{cm}^{3}$. The newly born $\mathrm{O}$ star will start to ionize the hydrogen in its surroundings. If the ionization could be assumed to happen instantaneously, the radius of the Strömgren sphere would become $r_{0}=s_{0} n_{0}^{-2 / 3}$.

Actually, the mass ionized per second may be found from equations (3), (16), and (19), taking the area $4 \pi r^{2}$ instead of $\pi R_{c}^{2}$, and $r_{0}$ instead of $s_{0}$. We obtain

$$
\frac{d M}{d t}=\frac{L_{u} m_{H}}{u}\left(1-\frac{r^{3}}{r_{0}^{3}}\right)=4 \pi g\left(1-\frac{r^{3}}{r_{0}^{3}}\right) \text {. }
$$

As an example we consider the propagation of the ionization front around an O5 star formed in a cloud with a density of 50 hydrogen atoms $/ \mathrm{cm}^{3}$. In this case $r_{0}$ is $13.6 \mathrm{pc}$. The speed of the ionization front is given by

$$
\frac{d r}{d t}=\frac{1}{4 \pi r^{2} n_{H} m_{H}} \frac{d M}{d t}
$$

For $r=0.5 r_{0}$, this becomes $1950 \mathrm{~km} / \mathrm{sec}$; for $r=0.9 r_{0}, 185 \mathrm{~km} / \mathrm{sec}$. These velocities are, respectively, two and one orders larger than the velocity of sound in the ionized mass. For all practical purposes the ionization may therefore be said to be instantaneous up to a distance very close to the radius of the Strömgren sphere. The same conclusion probably holds for all observed large emission nebulae. For the following discussion we shall assume that the ionization front coincides with the Strömgren sphere at all times during the development of a nebula. If the radius of the ionized sphere at an arbitrary time is called $r$, and its density $n$, we have, therefore,

$$
r=s_{0} n^{-2 / 3} \text {. }
$$

Let us assume that the dense nebula extends everywhere beyond the limit of the Strömgren sphere. The ionization by the newly born O star will increase the pressure in the inner part by a factor of about 100 over that in the cool surrounding mass. It has been shown elsewhere that, in actually observed nebulae, the gravitational forces are small compared to this pressure. As a consequence, the hot inner mass will expand into the cool shell. By the decrease in density in the inner mass, the radius of the Strömgren sphere will grow. It will grow faster than the radius of the original ionized mass, so that additional parts of the cool shell will become ionized. It would be outside the scope of the present article to attempt to discuss in detail the phenomena that will be caused by this expansion. We shall make two simplifying assumptions, which we hope will give a usable approximation for our very limited purpose. We assume that the ionized sphere remains homogeneous during the expansion. In order to have a concrete model, it will further be surmised that a compression region proceeds into the cool shell, probably with a velocity of the order of that of sound in the hot inner mass. ${ }^{2}$ It is not unlikely that the radiation will be sufficient to keep the compression region at a temperature

2 The case of an $H$ II region expanding into a neutral shell has been discussed along similar lines by Schatzman and Kahn (1953). They have paid more attention to the progress of the shock front, without considering the probably very important effects of cooling by radiation. 
comparable to that in the cool shell. The compression would then be approximately a hundred fold.

After the compression front has reached the outer surface of the nebula, the compressed shell will continue to expand. The expansion velocity will be determined by the pressure in the inner sphere as well as by the rocket effect of the matter ionized from the inner surface of the shell. In order to find the amount of acceleration, we shall need the mass of the neutral shell before and after the expansion. Let us denote these by $M_{0}^{(n)}$ and $M^{(n)}$, respectively. If, similarly, $M_{0}^{(i)}$ and $M^{(i)}$ are the masses of the ionized sphere before and after the expansion and $M$ is the total mass, we have, evidently,

$$
\frac{M^{(n)}}{M_{0}^{(n)}}=\frac{M-M^{(i)}}{M-M_{0}^{(i)}}=\frac{1-M^{(i)} M^{-1}}{1-M_{0}^{(i)} M^{-1}}
$$

Using equation (34), we can write

$$
M_{0}^{(i)}=\frac{4}{3} \pi n_{0} m_{H} r_{0}^{3}=\frac{4}{3} \pi m_{H} n_{0}^{-1} s_{0}^{3}
$$

and

$$
M^{(i)}=\frac{4}{3} \pi n m_{H} r^{3}=\frac{4}{3} \pi m_{H} s_{0}^{3 / 2} r^{3 / 2} .
$$

In the present section we are interested in very large and dense cloud complexes having densities between, say, 20 and 100 atoms $/ \mathrm{cm}^{3}$ and masses in excess of 20,000 solar masses. In these cases $M_{0}^{(i)}$ is rather smaller than $M$, as shown by the accompanying values of $M_{0}^{(i)}$. In the relevant cases the second term in the denominator of equation (35)

\begin{tabular}{c|r|r}
\hline \hline Sp. & $n_{H}=20$ & $n_{H}=100$ \\
\hline O5 $\ldots \ldots \ldots \ldots$ & 13,700 & 2,700 \\
O $7 \ldots \ldots \ldots$ & 3,300 & 660 \\
O9....... & 480 & 100 \\
\hline
\end{tabular}

will thus be small and may be omitted for the present rough estimates. If the original outer radius of the entire cloud is called $R$, we then obtain

$$
\frac{M^{(n)}}{M_{0}^{(n)}}=\frac{M^{(n)}}{M}=1-\frac{M^{(i)}}{M}=1-\frac{s_{0}^{3 / 2} r^{3 / 2}}{n_{0} R^{3}} .
$$

In this case the process of acceleration may well be limited by the sweeping-up of ordinary interstellar matter outside the cloud complex. If the average density of this matter is supposed to correspond to 1 hydrogen atom $/ \mathrm{cm}^{3}$, the amount swept up during an increase of the radius by $d r$ is $4 \pi m_{H} r^{2} d r$. The mass ionized in the same interval from the inner surface of the neutral shell is given by the differential of equation (37). The two are equal for

$$
r=2^{-2 / 3} s_{0}=0.63 s_{0} \text {. }
$$

The velocity of the shell will reach its maximum around this point.

In view of the chaotic conditions that we find in actual interstellar space, it would seem an adequate simplification to consider separately the effects of acceleration by the radiation of the $\mathrm{O}$ star and the slowing-down by sweeping up other clouds. Accordingly, we assume that, up to $r=0.5 s_{0}$, the collisions with other clouds are negligible and that beyond this distance the acceleration by the $O$ star's radiation is negligible.

On this basis we can compute the minimum mass that will escape total "evaporation" by the $\mathrm{O}$ star. This is equal to the mass within a Strömgren sphere having a radius $r=$ 
$\frac{1}{2} s_{0}$ and may be found by inserting this value of $r$ in equation (37). Expressed in the mass of the sun as unit we derive the minimum masses given in the accompanying table.

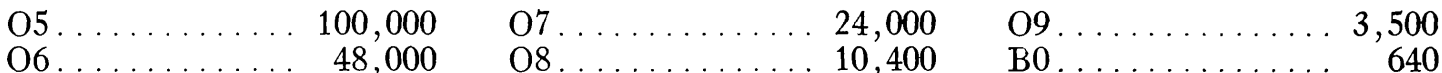

From the little that we know about the masses of large cloud complexes it seems probable that the masses of the larger ones are of the order of the first four numbers given in the table. The cloud complexes are always extremely uneven, so that probably in some parts the mass thickness will considerably exceed the limits required to escape total ionization, while in other directions they will be ionized completely. Owing to the irregularity, the neutral shell is likely to be broken up in the process of expansion, and we may imagine the various parts to form separate condensations in the interstellar matter that will resemble "ordinary" interstellar clouds. Through the expansion and evaporation process, these will be endowed with considerable velocity.

In order to obtain an estimate of this velocity, we combine equation (2) with equation (38). Again putting $r=0.5 s_{0}$, we find

$$
e^{-\left(v-v_{0}\right) / V}=1-\frac{0.35 s_{0}^{3}}{n_{0} R^{3}}
$$

In the discussion concerning $V$ given in Section III, it was tacitly supposed that the ionized particles escape into a practical vacuum. In the present case the inner sphere is filled with dense gas; yet the conditions resemble those discussed in Section III, because the compressed layer from which the "evaporation" takes place must have a density considerably exceeding that in the ionized inner mass. We do not know exactly what values to use for $v_{0}$ and $V$. We shall probably get too low values for $v$ if we assume $V=$ $20 \mathrm{~km} / \mathrm{sec}, v_{0}=0$. On the other hand, it seems unlikely that $V$ would have to be taken larger than $30 \mathrm{~km} / \mathrm{sec}$ and $v_{0}$ larger than $11 \mathrm{~km} / \mathrm{sec}$, the velocity of sound in the ionized sphere. We shall assume that $v$ lies between the extremes corresponding to these two suppositions.

Each of the large nebulae must put a considerable amount of kinetic energy into the interstellar medium. If the nebulae are large enough, much of this energy will be in the form of translational motion of compressed clouds. Very little is at present known about the masses of these large cloud complexes or about the frequency of their occurrence. In an article by Oort (1954) an estimate has been attempted. This indicated that the energy provided by the disruption of the large nebulae in the way discussed may well be of the right order to maintain the cloudy structure and the random motions in the interstellar medium.

\section{DISTRIBUTION OF CLOUD VELOCITIES}

As a working hypothesis we shall start from the assumption that the process just discussed is the principal cause of cloud motions. We may then inquire whether it can explain the observed distribution of high interstellar velocities.

To compute the entire velocity distribution would be an enormous labor. Even if sufficient data were available to make any such calculation, it would far surpass the scope of the present article. ${ }^{3}$ All we wish to do now is to see whether the mechanism could produce enough high velocities and whether the frequency and the distribution of the high velocities that would follow from it agree with those observed. In this discussion we shall once more use a drastic simplification. We shall assume that the clouds lose their high velocities when, after their acceleration, they collide for the first time with another cloud.

${ }^{3}$ Some calculations on the statistical effects of cloud collisions have been made by Kahn (1954b) and by Parker (1953). 
JAN H. OORT AND LYMAN SPITZER, JR.

The distribution law of interstellar velocities has recently been investigated by Blaauw (1952b), Searle (1952), and Schlüter, Schmidt, and Stumpff (1953), all investigations being based on data obtained by Adams (1949). Table 4, taken from a preliminary communication by Schlüter, Schmidt, and Stumpff, shows the observed distribution. The low-velocity part of the table has little significance, as it is greatly distorted by blending. The numbers of clouds with positive and negative velocities are given separately under $n^{+}$and $n^{-}$. All velocities were corrected for the effect of solar motion and differential galactic rotation. The moderately large velocities are preponderantly negative, as has been extensively commented upon by most authors (cf. the references given above). In accordance with our working hypothesis, we shall consider this as a consequence of the fact that the high-velocity clouds will often be observed against the background of the stars associated with the cloud complex from which they originated.

An individual inspection of the velocities in excess of $20 \mathrm{~km} / \mathrm{sec}$ already suffices to show that the large nebulae and the stellar associations which are probably directly related to them produce high-velocity clouds in the right order of frequency. For it appears that no less than two-thirds of the known residual velocities between 20 and 30 $\mathrm{km} / \mathrm{sec}$ are related to known stellar associations (Oort 1954).

TABLE 4

DISTRIBUTION OF INTERSTELLAR VELOCITIES

\begin{tabular}{c|r|r|r|r}
\hline $\begin{array}{c}v \\
(\mathrm{Km} / \mathrm{Sec})\end{array}$ & $n^{+}$ & $n^{-}$ & $n$ & $257 e^{-v / 12}$ \\
\hline$<10 \ldots \ldots$ & 143 & 164 & $(307)$ & $(145)$ \\
$10-20 \ldots \ldots$ & 21 & 37 & $58:$ & 65 \\
$20-30 \ldots \ldots$ & 4 & 30 & 34 & 27 \\
$30-70 \ldots \ldots$ & 7 & 10 & 17 & 20 \\
$>70 \ldots \ldots$ & 1 & 2 & 3 & 1 \\
\hline
\end{tabular}

The theory of the origin of high-velocity clouds worked out in the present article appears to fit satisfactorily the type of distribution observed for these high velocities. As may be seen from Table 4, we can fit the observed distribution well enough with a function of the form $e^{-v / \eta}$, where $\eta=12 \mathrm{~km} / \mathrm{sec}$. This is the same velocity law as was proposed by Blaauw (1952b) for all interstellar velocities; the value of $\eta$ adopted by Blaauw, however, was about $7 \mathrm{~km} / \mathrm{sec}$.

The way in which the velocity of a cloud escaping from the sphere of influence of an $\mathrm{O}$ star depends upon its original mass is given by equation (40) for the case of a large spherical cloud surrounding the $\mathrm{O}$ star, and by equation (23) for an isolated cloud. As the spherical shell will ultimately be broken up into separate parts, we shall in most cases have to deal with some kind of combination of equations (23) and (40). The velocity distribution is determined by the distribution of the masses of the original clouds.

The general character of the distribution law of high velocities that one would obtain is given in Table 5, which shows for one special case the velocities to which the clouds are accelerated. It refers to an $\mathrm{O} 5 \mathrm{star}$. For $v_{0}$ and $V$ we have used 11 and $20 \mathrm{~km} / \mathrm{sec}$, respectively. For the isolated cloud we assumed a constant radius of $5 \mathrm{pc}$ and $r_{0}=10 \mathrm{pc}$, while $r$ in equation (23) was taken equal to $s_{0}$, or $140 \mathrm{pc}$. In the case of the shell the original radius of the spherical cloud was taken to be $20 \mathrm{pc}$. The final velocities are tabulated as a function of $n_{0}$, the number of hydrogen atoms per cubic centimeter in the original cloud.

As the spherical shell will always break up into separate units, the case of the isolated 
cloud may be more representative. It is evident from a comparison of Tables 4 and 5 that the observed velocity distribution can be produced by a plausible distribution of $n_{0}$. The comparison applies only to the high velocities. Because of collisions during the later stages of a cloud's existence, there must, of course, be many more low velocities.

Moreover, examination of equations (23) and (40) shows that in each case the number of clouds of very high velocity varies asymptotically as $\exp (-v / V)$. Elsewhere in this paper we have set $V$ equal to $20 \mathrm{~km} / \mathrm{sec}$. It is readily shown that, for clouds of velocity $30 \mathrm{~km} / \mathrm{sec}$ or higher, this larger value of $V$ provides a better fit with Adams' data than is found with the value $12 \mathrm{~km} / \mathrm{sec}$ adopted in Table 4 .

TABLE 5

Final Velocity $v$ (IN KM/SEC) AS FunCtion OF Number OF HydRoGEN ATOMS PER $\mathrm{CM}^{3}\left(n_{0}\right)$ IN ORIGINAL CLOUD

\begin{tabular}{|c|c|c|c|c|c|}
\hline \multicolumn{3}{|c|}{ Isolated Cloud } & \multicolumn{3}{|c|}{ Spherical Shell } \\
\hline $\log n_{0}$ & $n_{0}$ & ข & $\log n_{0}$ & $n_{0}$ & $v$ \\
\hline $\begin{array}{l}2.00 \ldots \ldots \\
2.10 \ldots \ldots \\
2.20 \ldots \ldots \\
2.30 \ldots \ldots \\
2.60 \ldots \ldots \\
2.90 \ldots \ldots\end{array}$ & $\begin{array}{l}100 \\
126 \\
159 \\
200 \\
400 \\
800\end{array}$ & $\begin{array}{l}\infty \\
62 \\
46 \\
37 \\
24 \\
17\end{array}$ & $\begin{array}{l}2.079 \ldots \ldots \\
2.179 \ldots \ldots \\
2.280 \ldots \ldots \\
2.380 \ldots \\
2.681 \ldots \\
2.982 \ldots\end{array}$ & $\begin{array}{l}120 \\
151 \\
191 \\
240 \\
480 \\
960\end{array}$ & $\begin{array}{l}\infty \\
43 \\
31 \\
25 \\
17 \\
14\end{array}$ \\
\hline
\end{tabular}

\section{REFERENCES}

Adams, W. S. 1949, Ap. J., 109, 354.

Bates, D. R. 1950, Phys. Rev., 78, 492.

Beals, C. S. 1936, M.N., 96, 661.

Blaauw, A. 1952a, B.A.N., 11, 405 (No. 433).

- - $1952 b$, ibid., p. 459 (No. 436).

-—. 1954, A.J., 119, 625.

Blaauw, A., and Morgan, W. W. 1953, B.A.N., 12, 76 (No. 448).

Delhaye, J., and Blaauw, A. 1953, B.A.N., 12, 72 (No. 448).

Jentzsch; C., and Unsöld, A. 1948, Zs.f. Phys., 125, 370.

Kahn, F. D. 1954a, B.A.N., 12, 187 (No. 456). 1954b, M.N., 114 (in press).

Mayall, N.U. 1953, Pub. A.S.P., 65, 152.

Oort, J. H. 1952, Ap. J., 116, 233. . 1954, B.A.N., 12, 177 (No. 455).

Parker, E. 1953, Ap. J., 117, 169.

Schatzman, E., and Kahn, F. D. 1953, Paper read at Cambridge Symposium on Gas Dynamics of Interstellar Clouds.

Schlüter, A., Schmidt, H., and Stumpff, P. 1953, Zs.f. Ap., 33, 194.

Searle, L. T. 1952, Ap. J., 116, 650.

Spitzer, L., Jr. 1948a, Ap.J., 107, 6. $1948 b$, ibid., 108, 276.

Spitzer, L., Jr., and Savedoff, M. P. 1950, Ap. J., 111, 593.

Strömgren, B. 1939, Ap. J., 89, 526. .1948, Ap.J., 108, 242 .

Van de Hulst, H. C., Muller, C. A., and Oort, J. H. 1954, B.A.N., 12, 117 (No. 452).

Weizsäcker, C. F. von. 1948, Naturwiss., 35, 188.

. 1949, Problems of Cosmical Aerodynamics (Dayton, Ohio: Central Air Documents Office); Proceedings of the Paris Symposium on the Motion of Gaseous Masses of Cosmical Dimensions, chap. 22. Whipple, F. 1948, Centennial Symposia (Cambridge: Harvard College Observatory), sec. I, 8.

Wild, J. P. 1952, Ap. J., 115, 206. 